\title{
Some Aspects of Shear Yielding and Emergence of Shear Bands in Solid Polymers
}

\section{DoBOvŠEK*}

Faculty of Mathematics and Physics, University of Ljubljana, Jadranska 19, 1000 Ljubljana, Slovenia

\begin{abstract}
In this work, we first recapitulate experimental findings under different testing conditions available in the literature. Next, we establish a theoretical framework for the analysis of evolution of deformation into a highly localized pattern, where the theoretical approach with necessary adjustments to accommodate the constitutive behaviour of the solid polymer is applied in the course of development. A detailed theoretical analysis of critical stress conditions required for shear band initiation shows that in order to predict the material instability in accordance with experimental findings it is necessary to introduce a multi-parameter type of constitutive relation.
\end{abstract}

DOI: $10.12693 /$ APhysPolA.128.619

PACS: 46.35.+z, 83.10.Ff, 83.10.Gr, 83.80.Ab

\section{Introduction}

Experimental evidence has shown that there are two possible types of shear band behaviour in solid polymers. They are both triggered by shear yielding. The fine or the thin bands are more localized and are not significantly affected by normal stresses, while the coarse bands are highly influenced by the hydrostatic stress. From a theoretical perspective, shear bands represent a phenomenon of coexisting regions of a different strain rate. These regions are usually separated by sharp interfaces. They belong to a wider class of material instabilities that emerge as abrupt local changes in behaviour. Shear bands have been observed in various types of material media like granular materials, metals and plastics. It is now widely accepted that one of the most important triggering mechanisms for the onset of shear band is a relationship describing a non-monotonic stress and strain rate. During the process of deformation and viscoplastic flow such a relationship allows separation into two domains with different strain rates. This is understood to be the main reason for the onset of shear band formation, where homogeneous steady ground state becomes unstable and the system spatially separates into regions with low and high deformation rate. During the process, the traction and the traction rate remain continuous across the interface, which separates both domains. Consequently, the condition of traction and traction rate continuity across the band serves as a starting point in development of the corresponding shear band initiation criterion.

\section{Classification and shear band morphology}

There are essentially two qualitatively different material instability phenomena that can occur in solid polymers. The first one emanates from tensile yielding also known as crazing, and the second, on which we focus in

*e-mail: igor.dobovsek@fmf.uni-lj.si this work, namely shear yielding, results in shear band formation. Both belong to the category of localized deformation processes and are very important from the perspective of ductility and brittleness of polymeric materials. As we focus on shear banding phenomena, we first discuss mechanisms involved in the nucleation, multiplication, propagation and evolution of the shear bands.

Most semicrystalline polymers, particularly those with pronounced amorphous structure [1], develop two kinds of shear bands. The type of the band depends on the loading conditions and temperature. The first type, called the coarse band [2], occurs when the loading is fast at a normal low temperature. Here the important triggering mechanisms are stress concentrations. They propagate fast and are usually confined to a very thin localized area. Usually the process results in brittle failure along the band with moderate strain. In addition, the plane strain conditions near the sharp notches and thick sections define favourable conditions for this type of material instability behaviour.

In contrast to the first type, the second type appears when the loading is slow and the temperature is high. In this case, the shear zone consists of very fine bands, which spread on a larger region by mechanism of multiplication and ultimately end up in the form of ductile fracture at considerable total strain. This usually takes place after localized yielding and formation of a neck, which ultimately spreads throughout the cross-section of the specimen. The process leads to high strain gradients concentrated on a thin area under the plane stress conditions. In real system under the general operation conditions both types represent the complementary competing mechanisms responsible for formation of particular type of material instability.

The most distinctive feature of shear band behaviour in comparison with the tensile mode of instability in the form of crazing is shown under the compressive load conditions. Most glassy polymers undergo substantial plastic deformation at room temperature and moderate strain rates before the onset of fracture $[3,4]$. Deformation 
develops non-homogeneously where only local regions in the material are plastically deformed. Formation of intense and highly concentrated shear bands and diffuse shear zones depends on a variety of factors like specific loading conditions, ambient temperature, loading rate, morphology and molecular structure of the shear zones.

In the case of macroscopically inhomogeneous deformation, the qualitative understanding and quantitative evaluation of the phenomenon is much more complicated, and it is not completely developed from the theoretical point of view, due to the complexity of morphological mechanisms of structural deformation in a semicrystalline material.

\section{Shear yielding and structure of shear zones}

From experimental evidence, it is known that the coarse bands are affected by normal stress while the fine bands are not. Individual coarse shear bands appear under conditions of high-speed deformation and low temperature. Fine shear bands arranged in a broad diffuse shear zone originate under the low speed deformation and at higher temperatures. At the same time, the hydrostatic pressure effect is positive for the fine bands and negative for the coarse bands [5]. Under hydrostatic stress, polymer may have a higher yield stress, whereas its ductility decreases in compression.

The evolution of shear bands consists of two stages. Usually the shorter bands are formed first, which later on intersect with larger shear bands that have been triggered by a notch in the specimen or by some other originator of stress concentration within the specimen.

In compression tests, it has been observed that shear bands evolve and spread under angles within the range between 35 to 45 degrees with respect to the compressive axis [6]. This is a generic range for the shear band inclination in broader family of glassy polymers and amorphous metals. Another important experimental observation is the band thickness, which is of the order of one $\mu \mathrm{m}$ in magnitude. Experimental measurements based on transmission electron microscopy (TEM) micrographs showed that the deformation is concentrated in localized areas of the specimen in the so-called discontinuous deformation zones with the thickness in the range between $0.1 \mu \mathrm{m}$ to $0.5 \mu \mathrm{m}$.

Temperature dependence is also very important feature in the shear band evolution. The higher the ambient temperature, the higher the spatial density of coarse shear bands. If the testing temperature is near the glass transition temperature, usually a broad shear zone develops at an angle of 45 degrees to the principal stress direction. The ratio of ambient temperature versus glass transition temperature to a large extent determines the material instability deformation mode. Under shear stress conditions, the ratio indicates whether the coarse shear band will form, or whether deformation will end up in the diffuse shear zone mode. If the ratio is about 0.75 , coarse shear bands or homogeneous shear zone can occur, depending on a strain rate. For ratios above 1 diffuse shear zone becomes more probable.

\section{The constitutive model}

Because the strain localization and shear banding phenomena are closely related to the combined action of nonlinear plastic flow, strain hardening, and intrinsic strain softening, the mathematical model should be general enough to accommodate such behaviour. To be able to describe in mathematical terms the shear banding phenomena in solid polymers, a multi-parameter constitutive relation is used, which connects the critical shear stress, a hydrostatic part of stress tensor, pressure sensitivity, and a special evolution parameter in the form of strain softening. The constitutive model is based on a general framework of nonlinear viscoelastic flow [7].

In the sequel, we give a brief outline of a general theory. A spatial point in $\mathbb{R}^{3}$ is represented by a position vector $\boldsymbol{x}$ with respect to fixed orthonormal basis $\left\{\widehat{\boldsymbol{e}}_{1}, \widehat{\boldsymbol{e}}_{2}, \widehat{\boldsymbol{e}}_{3}\right\}, \widehat{\boldsymbol{e}}_{i} \cdot \widehat{\boldsymbol{e}}_{j}=\delta_{i j}, \boldsymbol{I}=\delta_{i j} \widehat{\boldsymbol{e}}_{i} \otimes \widehat{\boldsymbol{e}}_{j}, \boldsymbol{v}(x, t)$ is the velocity at a spatial point $\boldsymbol{x}$ at a given instant of time $t$. In description of three-dimensional deformation of solid polymers, we use the Maxwell co-rotational model, which serves as a starting point in further development. In this model it is assumed that the velocity gradient is decomposed in an elastic and a plastic part with its symmetric ( $\boldsymbol{D}$ rate of deformation tensor) and skew symmetric ( $\boldsymbol{W}$ spin tensor) counterparts

$$
\boldsymbol{L}=\boldsymbol{L}_{\mathrm{E}}+\boldsymbol{L}_{\mathrm{P}}=\left(\boldsymbol{D}_{\mathrm{E}}+\boldsymbol{W}_{\mathrm{E}}\right)+\left(\boldsymbol{D}_{\mathrm{P}}+\boldsymbol{W}_{\mathrm{P}}\right) .
$$

In what follows, we employ the multiparameter generalization of the Eyring viscosity $[8,9] \eta=\eta(p, \bar{\tau}, \beta, s, T)$, where $p$ is the pressure, $\beta$ accounts for pressure dependence, $\bar{\tau}$ is the effective stress, $s$ is special evolution parameter describing strain softening, and $T$ is the absolute temperature. According to the well known decompositions

$$
\begin{aligned}
& \boldsymbol{\sigma}=\operatorname{dev}(\boldsymbol{\sigma})+\frac{1}{3}(\operatorname{Tr} \boldsymbol{\sigma}) \boldsymbol{I}=\boldsymbol{\sigma}^{\prime}-p \boldsymbol{I}, p=-\frac{1}{3} \operatorname{Tr} \boldsymbol{\sigma}, \\
& \bar{\tau}=\sqrt{\operatorname{Tr}\left(\boldsymbol{\sigma}^{\prime} \boldsymbol{\sigma}^{\prime}\right) / 2}, \\
& \boldsymbol{D}^{\prime}=\operatorname{dev}(\boldsymbol{D})=\boldsymbol{D}-\frac{1}{3} \operatorname{Tr}(\boldsymbol{D}) \boldsymbol{I}, \\
& \dot{\bar{\gamma}}_{\mathrm{P}}=\sqrt{2 \operatorname{Tr}\left(\mathrm{D}_{\mathrm{P}} \mathrm{D}_{\mathrm{P}}\right)},
\end{aligned}
$$

in the sequel, we also neglect the volumetric part of the plastic deformation in the constitutive equation, so that the deviatoric part of the Cauchy true stress $\boldsymbol{\sigma}$ can be connected with the plastic part of rate of deformation tensor as

$$
\boldsymbol{\sigma}^{\prime}=2 \eta(p, \bar{\tau}, \beta, s, T) \boldsymbol{D}_{\mathrm{P}}
$$

The elastic part of deformation is modelled by the hypoelastic rate formulation employing the Hooke law for isotropic materials, so that the objective time derivative of the true stress and the elastic part of strain of deformation tensor can be written as

$$
\begin{gathered}
\stackrel{\nabla}{\boldsymbol{\sigma}}=\lambda \operatorname{Tr}\left(\boldsymbol{D}_{\mathrm{E}}\right) \boldsymbol{I}+2 \mu \boldsymbol{D}_{\mathrm{E}}= \\
\boldsymbol{L}_{\mathrm{E}}: \boldsymbol{D}_{\mathrm{E}}=\boldsymbol{L}_{\mathrm{E}}:\left(D-D_{\mathrm{P}}\right),
\end{gathered}
$$

where $\boldsymbol{\lambda}$ and $\boldsymbol{\mu}$ are the Lame constants and the objective time derivative of true stress is represented by the Jaumann rate 


$$
\begin{aligned}
& \stackrel{\nabla}{\boldsymbol{\sigma}}=\dot{\boldsymbol{\sigma}}-\boldsymbol{W} \cdot \boldsymbol{\sigma}-\boldsymbol{\sigma} \cdot \boldsymbol{W}^{\top}=\dot{\boldsymbol{\sigma}}+\boldsymbol{\Omega}: \boldsymbol{L}= \\
& \left(\dot{\boldsymbol{\sigma}}_{i j}+\boldsymbol{\Omega}_{i j k l} L_{k l}\right) \widehat{\boldsymbol{e}}_{i} \otimes \widehat{\boldsymbol{e}}_{j}, \\
& \boldsymbol{\Omega}=\frac{1}{2}\left(T_{i k} \delta_{j l}-T_{j l} \delta_{i k}+T_{j k} \delta_{i l}-T_{i l} \delta_{j k}\right) \\
& \times \widehat{\boldsymbol{e}}_{i} \otimes \widehat{\boldsymbol{e}}_{j} \otimes \widehat{\boldsymbol{e}}_{k} \otimes \widehat{\boldsymbol{e}}_{l} .
\end{aligned}
$$

Combination among formerly defined relations leads to the co-rotational Maxwell model

$$
\stackrel{\nabla}{\boldsymbol{\sigma}}+\chi \boldsymbol{\sigma}^{\prime}=\lambda \operatorname{Tr}(\boldsymbol{D}) \boldsymbol{I}+2 \mu \boldsymbol{D}, \quad \chi=\mu / \eta .
$$

Another alternative in mathematical description of material behaviour of solid polymers with semi-crystalline and amorphous structure is a compressible Leonov model with formal decoupling of deformation for change in volume and isochoric response for change in shape of the specimen. Here, the free energy of the system is determined by two state variables, namely the relative volumetric deformation $J=\operatorname{det}(\boldsymbol{F})$, where $\boldsymbol{F}$ is the deformation gradient, and the isochoric (volume preserving) left Cauchy-Green strain tensor $\boldsymbol{B}_{\mathrm{E}}$ with $\operatorname{det}\left(\boldsymbol{B}_{\mathrm{E}}\right)=1$. Such a decomposition results in hydrostatic stress coupling with the volumetric deformation, whereas the deviatoric stress is determined by the isochoric part of elastic strain. For small volume deformations the compressible Leonov model reduces to

$$
\begin{aligned}
& \boldsymbol{\sigma}=\kappa(J-1) \boldsymbol{I}+\mu \boldsymbol{B}_{\mathrm{E}}^{\prime}, \\
& \stackrel{\nabla}{\boldsymbol{B}}_{\mathrm{E}}=\left(\boldsymbol{D}^{\prime}-\boldsymbol{D}_{\mathrm{P}}\right) \cdot \boldsymbol{B}_{\mathrm{E}}+\boldsymbol{B}_{\mathrm{E}} \cdot\left(\boldsymbol{D}^{\prime}-\boldsymbol{D}_{\mathrm{P}}\right), \\
& \dot{J}=J \operatorname{Tr}(\boldsymbol{D}), \quad \boldsymbol{\sigma}^{\prime}=2 \eta \boldsymbol{D}_{\mathrm{P}},
\end{aligned}
$$

where $\kappa$ is the bulk modulus, $\mu$ is the shear modulus. The Jaumann co-rotational derivative of the isochoric left Cauchy-Green strain tensor expressed in terms of the vorticity tensor is given as

$$
\stackrel{\nabla}{B}_{\mathrm{E}}=\dot{\boldsymbol{B}}_{\mathrm{E}}-\boldsymbol{W} \cdot \boldsymbol{B}_{\mathrm{E}}-\boldsymbol{B}_{\mathrm{E}} \cdot \boldsymbol{W}^{\top} \text {. }
$$

The Leonov model efficiently describes separation of the elastic hydrostatic and viscoelastic deviatoric stress response and consistently depicts the yield behaviour of solid polymers, where the plastic strain rate is described by the stress activated Eyring flow influenced by the strain softening and pressure sensitivity and exhibits the same mathematical dependence on material parameters as previously defined viscosity function. It is a matter of simple exercise to show that in the limit of small elastic deformations both models are equivalent under the same set of simplifying conditions. To prove that, one can take the co-rotational derivative of the stress in Eq. (7), substitute the evolution equation for $J$ and $\boldsymbol{B}_{\mathrm{E}}$ for small isochoric elastic an volumetric strains, and after some manipulation obtain the co-rotational Maxwell model given by Eq. (6). Consequently, both models essentially describe the same kinematics of deformation and evolution of true stress. In recent works, certain authors [10-12] have tried to adjust and generalize the concept of a nonNewtonian flow rule with the Eyring viscosity to account for more diversified constitutive response under different flow conditions. Result of past research is the viscosity function of the form

$$
\eta(p, \bar{\tau}, \beta, s, T)=A_{0}(T) \tau_{0} \exp \left(\frac{\beta p}{\tau_{0}}-s\right) \frac{\bar{\tau} / \tau_{0}}{\sinh \left(\bar{\tau} / \tau_{0}\right)},
$$

where the temperature dependent factor $A_{0}(T)$ and the characteristic stress $\tau_{0}$ are defined as

$$
A_{0}(T)=A_{0} \exp \left(\frac{\Delta U}{R T}\right), \quad \tau_{0}=\frac{k T}{V} .
$$

Here $A_{0}$ is a constant, $\Delta U$ is the activation energy, $R$ is the gas constant, $V$ is the activation volume, and $k$ is the Boltzmann constant. Same authors describe strain softening by means of an evolution equation

$$
\dot{s}=\dot{s}_{0}\left(1-\frac{s}{s_{\infty}}\right) \dot{\bar{\gamma}}_{\mathrm{P}}
$$

where $\dot{s}_{0}$ determines the initial softening rate, with initial value $s_{0}$ and an equilibrium value $s_{\infty}$ influenced by the equivalent plastic strain rate $\dot{\bar{\gamma}}_{\mathrm{P}}$.

\section{Shear band initiation criterion}

In what follows we use the co-rotational Maxwell model as a prototype constitutive relation for mathematical formulation of the shear band initiation criterion. Let the total stress $\sigma^{0}$ and the velocity gradient $L^{0}$ both resolved with respect to the fixed orthonormal basis as

$$
\boldsymbol{\sigma}^{0}=\boldsymbol{\sigma}_{i j}^{0} \widehat{\boldsymbol{e}}_{i} \otimes \widehat{\boldsymbol{e}}_{j}, \quad L^{0}=\left[\frac{\partial v}{\partial x}\right]_{0}=\left[\frac{\partial v_{i}}{\partial x_{j}}\right]_{0} \widehat{\boldsymbol{e}}_{i} \otimes \widehat{\boldsymbol{e}}_{j},
$$

represent spatially homogeneous fields. Introduce a rank one type of perturbation of velocity gradient over the band of a thin surface - a singular material surface in the limit of zero thickness. The jump operator is defined as $\llbracket \cdot \rrbracket=($ in $)-($ out $)=(+)-(-)$, so that the corresponding jump in velocity gradient across the shear band together with the jumps in its symmetric and antisymmetric part representing the rate of deformation tensor and the spin tensor, respectively, can be written as

$$
\begin{aligned}
& \llbracket \boldsymbol{L} \rrbracket=\boldsymbol{L}^{+}-\boldsymbol{L}^{-}=\llbracket \frac{\partial \boldsymbol{v}}{\partial x} \rrbracket=\boldsymbol{g} \otimes \boldsymbol{n}, \\
& \llbracket \boldsymbol{D} \rrbracket=\frac{1}{2}(\boldsymbol{g} \otimes \boldsymbol{n}+\boldsymbol{n} \otimes \boldsymbol{g}), \llbracket \boldsymbol{W} \rrbracket=\frac{1}{2}(\boldsymbol{g} \otimes \boldsymbol{n}-\boldsymbol{n} \otimes \boldsymbol{g}),
\end{aligned}
$$

where $\boldsymbol{g}$ designates amplitude of the jump, and $\boldsymbol{n}$ represents a normal to the band. Qualitative illustration of the system is depicted in Fig. 1. By invoking the starting assumption about homogeneity of the fields $\boldsymbol{\sigma}^{0}$ and $\boldsymbol{L}^{0}$ it can be shown that in addition to traction continuity across the band the corresponding traction rate continuity has to be satisfied as well. Now apply the corresponding jump operator $\llbracket \cdot \rrbracket$ across the shear band as a rank one type of perturbation of velocity gradient, with kinematics defined in Eq. (13), on Eqs. (5) and (6) and obtain

$$
\begin{aligned}
& \llbracket \dot{\boldsymbol{\sigma}} \rrbracket+\boldsymbol{\Omega}: \boldsymbol{g} \otimes \boldsymbol{n}+\llbracket \chi \rrbracket \boldsymbol{\sigma}^{\prime}= \\
& \lambda(\boldsymbol{g} \cdot \boldsymbol{n}) \boldsymbol{I}+\mu(\boldsymbol{g} \otimes \boldsymbol{n}+\boldsymbol{n} \otimes \boldsymbol{g}) .
\end{aligned}
$$

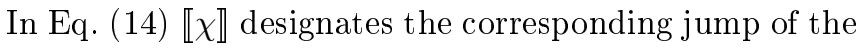
material parameter involving generalized viscosity. From conditions for traction continuity $n \cdot \llbracket \boldsymbol{\sigma} \rrbracket=\mathbf{0}$ and traction 
rate continuity $\boldsymbol{n} \cdot \llbracket \dot{\boldsymbol{\sigma}} \rrbracket=\mathbf{0}$, which have to be fulfilled across the shear band during the flow process, we arrive at

$$
\boldsymbol{Q} \cdot \boldsymbol{g}=\llbracket \chi \rrbracket p \boldsymbol{n},
$$

where $Q$ is given in the following form:

$$
\begin{aligned}
\boldsymbol{Q} & =\left(\mu+\frac{1}{2} \boldsymbol{n} \cdot \boldsymbol{\sigma} \cdot \boldsymbol{n}\right) \boldsymbol{I}+(\lambda+\mu) \boldsymbol{n} \otimes \boldsymbol{n} \\
& +\frac{1}{2}(\boldsymbol{t} \otimes \boldsymbol{n}-\boldsymbol{n} \otimes \boldsymbol{t})-\frac{1}{2} \boldsymbol{\sigma} .
\end{aligned}
$$

Here $\boldsymbol{t}=\boldsymbol{n} \cdot \boldsymbol{\sigma}$ represents a stress traction exerted on

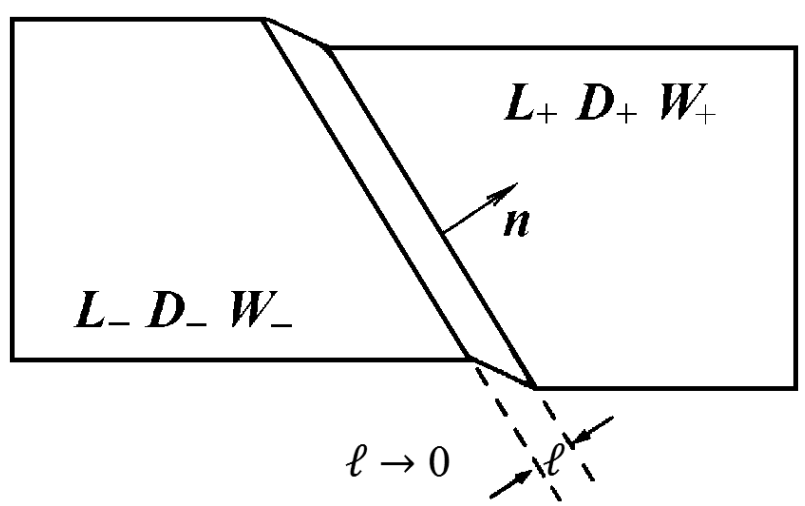

Fig. 1. Geometry and kinematics of deformation across the shear band.

the shear band. In Eqs. (14), (15) and (16) kinematics of deformation, stress fields, as well as constitutive variables, pertain to the homogeneous state outside the band. These fields were originally designated with the sign (-) which is omitted from the notation in the sequel. Equation (15) defines a relationship among vectors of amplitude jump $\boldsymbol{g}$, orientation of the band defined by the normal vector $\boldsymbol{n}$, current state of stress, and the constitutive parameters of the substance. In principle, the operator $\boldsymbol{Q}$ can be inverted, providing the solution for the amplitude jump $\boldsymbol{g}$ across the shear band. Calculation requires a careful inspection of potential finite jump discontinuities among material parameters and related fields in the expression for the material constitutive function including generalized viscosity. It is important to emphasize that such an analysis should be made on the micromechanical basis. The morphological restructuring in the shear band induced by deformation instability strongly depends on local material properties. During the process of deformation initial material morphology is transformed by yield into oriented fibrous microstructure. The process consists of several stages beginning with the separation, tilting, shearing, fragmentation, and finally terminates in a fibre-bundle like orientation. In the first stage, the continuous plastic deformation takes place before phenomenon of necking sets in. During the initial stage several elementary micromechanical mechanisms occur, like rotation of lamellar structure, chain slip, and tilt inside crystalline lamellae. In the second stage, the discontinuous transformation in the neck from the lamellar to the fibrous structure takes place. In the third stage, the plastic deformation of the fibre structure becomes predominant and leads to micro sliding of fibrils relative to each other resulting in the well-formed shear band. Needless to say, the mathematical description of the process on a micromechanical level is extremely difficult.

On the other hand, the macroscopic framework of the problem is well-developed. Additional analytical aspects from a broader perspective of material instability in rate dependent solids, which are required for determination of the critical conditions for the onset of material instability, are discussed in [13]. A general approach with details of derivation of shear band initiation criterion for certain classes of nonlinear viscoelastic flow can be found in [14].

\section{Final remarks}

Shear bands can occur under applied tensile or compressive stress. The process is triggered and controlled by shear stress, which induces the lateral slip movements of the material without void formation. Once the shear band has formed, the further continuing deformation concentrates within the thin band where the strain gradient continues to grow and remains confined in the narrow localized zone of the material. During the process of deformation, the local increase of the temperature within the localized zone can be detected. This has been confirmed in some experimental and theoretical studies [15], where the relationship among strain softening, adiabatic heating and strain rate sensitivity at the onset of criticality has been analysed in detail. Interactions among pertinent fields on the critical level can be perceived as a mathematical description of a precursor of unstable deformation in the form of localized shear. However, pure phenomenology does not provide a complete answer to the shear band initiation problem. The main problem in creating an adequate mathematical model is the difficulty of establishing a tractable connection between the macro- and microscale via the Eyring function formalism outside the shear band and the corresponding jump of the function across the shear band. This requires fundamental understanding of deformation mechanisms on a microscale. To link the phenomenology of the macroscale with complex morphology of the microscale, we need to extend the fundamental set of variables in constitutive relation and proceed from there. For this reason, it is feasible to introduce an additional geometric object representing a configuration structure within the band. Research in regard to creating the corresponding multiscale model is at the early stage of development.

\section{References}

[1] J. Perez, Physics and Mechanics of Amorphous Polymers, Balkema, Rotterdam 1998.

[2] C.C. Chau, J.C.M. Li, J. Mater. Sci. 14, 1593 (1979).

[3] I.M. Ward, Mechanical Properties of Solid Polymers, 2nd ed., Wiley, Chichester 1985. 
[4] R.A. Duckett, in: Structure and Properties of Oriented Polymers, Ed. I.M. Ward, Wiley, New York 1975 , p. 366.

[5] J.C.M. Li, J.B.C. Wu, J. Mater. Sci. 11, 445 (1976).

[6] C.C. Chau, J.C.M. Li, J. Mater. Sci. 14, 2172 (1979).

[7] F.J. Lockett, Nonlinear Viscoelastic Solids, Academic Press, London 1972.

[8] J.D. Ferry, Viscoelastic Properties of Polymers, 3rd ed., Wiley, New York 1980.

[9] J.M. Dealy, R.G. Larson, Structure and Rheology of Molten Polymers, Hanser Publ., Munich 2006.
[10] M.C. Boyce, D.M. Parks, A.S. Argon, Mech. Mater. 7, 15 (1988).

[11] P.D. Wu, E. van der Giessen, Int. J. Solids Struct. 31, 1493 (1994).

[12] T.A. Tervoort, R.J.M. Smith, W.A.M. Brekelmans, L.E. Govaert, Mech. Time-Depend. Mater. 1, 269 (1998).

[13] I. Dobovšek, B. Moran, Eur. J. Mech. A-Solid 15, 267 (1996).

[14] I. Dobovšek, AIP Conf. Proc. 1027, 300 (2008).

[15] I. Dobovšek, Arch. Mech. 46, 893 (1994). 